\title{
Retrial Queuing System with Starting Failure, Single Vacation and Orbital Search
}

\author{
D. Sumitha \\ Asst. Professor (SS) \\ Avinashilingam University, \\ Coimbatore
}

\author{
K. Udaya Chandrika \\ Professor \\ Avinashilingam University, \\ Coimbatore
}

\begin{abstract}
This paper is concerned with the analysis of a single server retrial queue with vacation and orbital search. The server is subject to starting failure and repair. At the completion epoch of each service, the server may take a single vacation. After vacation completion, the server searches for the customers in the orbit or remains idle. Retrial times, service times and vacation times are assumed to be arbitrarily distributed. Various performance measures are derived and numerical results are presented.
\end{abstract}

\section{INTRODUCTION}

Vacation models had been the subject of interest to queue theorists of deep study in recent years because of their applicability and theoretical structures in real life congestion situations such as manufacturing and production, computer and communication systems, service and distribution systems, etc. The most remarkable work done by some researchers on vacation models include Doshi (1990), Takagi (1991), Ke (2003), Baba (2005) and Wu and Takagi (2005).

One of the most important characteristic in the service facility of a queueing system is its starting failures. An arriving customer who finds the server idle must turn on the server. If the server is started successfully the customer gets the service immediately. Otherwise the repair for the server begins and the customer must join the orbit. The server is assumed to be reliable during service. Such systems with starting failures have been studied as queueing models by Yang and Li (1994), Krishna kumar et al. (2002), Mokaddis et al. (2007) and Ke and Chang (2009).

Retrial Queues considered by researchers so far have the characteristic that each service is preceded and followed by an idle period. Artalejo et al. (2002) have considered a retrial queue in which immediately after a service completion the server searches for customer from the orbit or remains idle. If a search is made a service is followed by another service, and if not, a service is followed by an idle time. Research work on retrial queueing system with orbital search is seldom found in literature. This motivates to study a single server retrial queue with vacation, starting failure and orbital search.

\section{MODEL DESCRIPTION}

Consider a single server retrial queue with the server subject to starting failure. Customers arrive at the system according to Poisson process with rate $\lambda$. If the server is found to be busy the arriving customers enter the retrial queue to try again for their requests after random intervals. Successive interretrial times of any customer are governed by an arbitrary probability distribution function $A(x)$ and Laplace-Stieltjes transform $A^{*}(s)$. If the server is free, an arriving customer must start the server, which takes negligible time. If the server is started successfully, the customer gets service immediately. Otherwise the repair for the server commences immediately and the customer must leave for the orbit and make a retrial at a later time. Successive repair times are independent and identically distributed with probability distribution function $G(x)$, Laplace-Stieltjes transform $G^{*}(s)$ and the first two moments $g_{1}$ and $g_{2}$. The probability of successful commencement of service is assumed to be $\alpha$.

Successive service times are independent with common probability distribution function $B(x)$, Laplace-Stieltjes transform $B^{*}(s)$ and the first two moments $b_{1}$ and $b_{2}$. At the completion of each service the server may take a vacation with probability $\beta$ or waits for the next customer with complementary probability $\delta$. After completing vacation, the server searches for customer in the orbit (if any) with probability $\theta$ or remains idle with probability $\theta(=1-\theta)$. The search time is assumed to be negligible. Vacation times are general with common probability distribution function $V(x)$, Laplace-Stieltjes transform $V^{*}(s)$ and the first two moments $v_{1}$ and $v_{2}$.

The state of the system at time $t$ can be described by the Markov process, $\{\mathrm{N}(\mathrm{t}) ; \mathrm{t} \geq 0\}=\left\{\mathrm{C}(\mathrm{t}), \mathrm{X}(\mathrm{t}), \xi_{0}(\mathrm{t}), \xi_{1}(\mathrm{t}), \xi_{2}(\mathrm{t})\right.$, $\left.\xi_{3}(t), t \geq 0\right\}$ where $\mathrm{C}(\mathrm{t})$ denotes the server state $0,1,2$ or 3 according as the server being free, providing the service, under repair or on vacation respectively and $X(\mathrm{t})$ corresponds to the number of customers in the orbit at time t. If $\mathrm{C}(\mathrm{t})=0$ and $X(t)>0$ then $\xi_{0}(t)$ represents the elapsed retrial time, if $\mathrm{C}(\mathrm{t})=1$ and $\mathrm{X}(\mathrm{t})>0$ then $\xi_{1}(\mathrm{t})$ corresponds to the elapsed time of the customer being provided service, if $\mathrm{C}(\mathrm{t})=2$ and $\mathrm{X}(\mathrm{t})>0$ then $\xi_{2}(\mathrm{t})$ represents the elapsed repair time and if $\mathrm{C}(\mathrm{t})=3$ and $\mathrm{X}(\mathrm{t})>0$ then $\xi_{3}(\mathrm{t})$ represents the elapsed vacation time at time $\mathrm{t}$.

Let the functions $\eta(\mathrm{x}), \mu(\mathrm{x}), \mathrm{r}(\mathrm{x})$ and $\gamma(\mathrm{x})$ be the conditional completion rates at time $\mathrm{x}$ respectively for repeated attempts, service, repair and vacation. Then

$$
\begin{aligned}
& \eta(x)=a(x) /(1-A(x)) ; \mu(x)=b(x) /(1-B(x)) \\
& r(x)=g(x) /(1-G(x)) \text { and } \gamma(x)=v(x) /(1-V(x)) .
\end{aligned}
$$




\section{STABILITY CONDITION}

The following theorem provides the necessary and sufficient condition for the system to be stable.

\section{Theorem 1.}

The embedded Markov chain $\left\{\mathrm{N}_{\mathrm{n}}, \mathrm{n} \in \mathrm{N}\right\}$ is ergodic if and only if

$$
\lambda\left(\alpha \beta v_{1}+\alpha b_{1}+\bar{\alpha} g_{1}(1-\theta \beta)\right)+\bar{\alpha}<A *(\lambda)+\theta \beta\left(1-A^{*}(\lambda)\right) .
$$

\section{Proof:}

$\left\{N_{n}, n \in N\right\}$ is an irreducible aperiodic Markov chain. To prove ergodicity, we shall use the Foster's criterion, an irreducible and aperiodic Markov chain is ergodic if there exists a non negative function $f(j), j \in N$ and $\xi>0$ such that the mean drift $x_{j}=E\left[f\left(N_{n+1}\right)-f\left(N_{n}\right) I N_{n}=j\right]$ is finite for all $j \epsilon$ $\mathrm{N}$ and $\mathrm{x}_{\mathrm{j}} \leq-\xi$ for all $\mathrm{j} \epsilon \mathrm{N}$ except for a finite number of $j \mathrm{~s}$. Here we consider the function $f(j)=j$. Then we have $\mathrm{x}_{\mathrm{j}}=$

$$
\begin{aligned}
& \mathrm{x}_{\mathrm{j}}= \\
& \left\{\begin{array}{l}
\lambda\left(\alpha \beta v_{1}+\alpha b_{1}+\bar{\alpha} g_{1}(1-\theta \beta)\right)+\bar{\alpha}-A^{*}(\lambda)-\theta \beta\left(1-A^{*}(\lambda)\right), j=1,2,3 \ldots \ldots . . \\
\mathrm{E}(\mathrm{x}), \mathrm{j}=0
\end{array}\right.
\end{aligned}
$$

Clearly the inequality $\lambda\left(\alpha \beta v_{1}+\alpha b_{1}+\alpha g_{1}(1-\theta \beta)\right)+\bar{\alpha}<A *(\lambda)+\theta \beta\left(1-A^{*}(\lambda)\right)$ is $\quad$ a sufficient condition for ergodicity.

The same inequality is also necessary for ergodicity. As noted in Sennot et al. (1983), we can guarantee non ergodicity, if the Markov chain $\left\{\mathrm{N}_{\mathrm{n}}, \mathrm{n} \in \mathrm{N}\right\}$ satisfies Kaplan's condition, namely $x_{j}<\infty$ for all $j \geq 0$ and there exists $j_{0} \in N$ such that $\mathrm{x}_{\mathrm{j}} \geq 0$ and $\mathrm{j} \geq \mathrm{j}_{0}$. In our case Kaplan's condition is satisfied because there is $k \in \mathrm{N}$ such that $\mathrm{r}_{\mathrm{ij}}=0$ for $\mathrm{j}<\mathrm{i}-\mathrm{k}$ and $\mathrm{i}>0$, where $\left(\mathrm{r}_{\mathrm{ij}}\right)$ is the one step transition matrix of $\left\{\mathrm{N}_{\mathrm{n}}, \mathrm{n} \in \mathrm{N}\right\}$. Then

$\lambda\left(\alpha \beta v_{1}+\alpha b_{1}+\bar{\alpha} g_{1}(1-\theta \beta)\right)+\bar{\alpha}>A^{*}(\lambda)+\theta \beta\left(1-A^{*}(\lambda)\right)$ implies the non ergodicity of the Markov chain.

\section{STEADY STATE DISTRIBUTION}

\section{Define}

$I_{0}(t)=P\{C(t)=0, X(t)=0\}$

$I_{n}(x, t) d x=P\left\{C(t)=0, X(t)=n, x \leq \xi_{0}(t)<x+d x\right\} ;$

$$
t \geq 0, x \geq 0, n \geq 1
$$

$W_{n}(x, t) d x=P\left\{C(t)=1, X(t)=n, x \leq \xi_{1}(t)<x+d x\right\}$;

$$
t \geq 0, x \geq 0, n \geq 0
$$

$R_{n}(x, t) d x=P\left\{C(t)=2, X(t)=n, x \leq \xi_{2}(t)<x+d x\right\}$;

$$
t \geq 0, x \geq 0, n \geq 1
$$

$V_{n}(x, t) d x=P\left\{C(t)=3, X(t)=n, x \leq \xi_{3}(t)<x+d x\right\}$;

$$
t \geq 0, x \geq 0, n \geq 0
$$

The system of steady state equations that governs the model is given below

$$
\lambda I_{0}=(1-\beta) \int_{0}^{\infty} W_{0}(x) \mu(x) d x+\int_{0}^{\infty} V_{0}(x) \gamma(x) d x
$$

$$
\begin{aligned}
& \frac{d}{d x} I_{n}(x)=-(\lambda+\eta(x)) I_{n}(x), n \geq 1 \\
& \frac{d}{d x} W_{0}(x)=-(\lambda+\mu(x)) W_{0}(x) \\
& \frac{d}{d x} W_{n}(x)=-(\lambda+\mu(x)) W_{n}(x)+\lambda W_{n-1}(x), n \geq 1 \\
& \frac{d}{d x} R_{1}(x)=-(\lambda+r(x)) R_{1}(x) \\
& \frac{d}{d x} R_{n}(x)=-(\lambda+r(x)) R_{n}(x)+\lambda R_{n-1}(x), n \geq 2 \\
& \frac{d}{d x} V_{0}(x)=-(\lambda+\gamma(x)) V_{0}(x) \\
& \frac{d}{d x} V_{n}(x)=-(\lambda+\gamma(x)) V_{n}(x)+\lambda V_{n-1}(x), n \geq 1
\end{aligned}
$$

with boundary conditions

$$
\begin{aligned}
I_{0}(0)= & (1-\beta) \int_{0}^{\infty} W_{n}(x) \mu(x) d x+(1-\theta) \int_{0}^{\infty} V_{n}(x) \gamma(x) d x \\
& +\int_{0}^{\infty} R_{n}(x) r(x) d x, n \geq 1 \\
W_{0}(0)= & \alpha \lambda I_{0}+\alpha \int_{0}^{\infty} I_{1}(x) \eta(x) d x+\theta \int_{0}^{\infty} V_{1}(x) \gamma(x) d x \\
W_{n}(0)= & \alpha \lambda \int_{0}^{\infty} I_{n}(x) d x+\alpha \int_{0}^{\infty} I_{n+1}(x) \eta(x) d x+ \\
& \quad \theta \int_{0}^{\infty} V_{n+1}(x) \gamma(x) d x, n \geq 1 \\
& -\alpha \lambda I_{0}+\bar{\alpha} \int_{0}^{\infty} I_{1}(x) \eta(x) d x \\
R_{1}(0)= & -\alpha \lambda \int_{0}^{\infty} I_{n-1}(x) \eta(x) d x+\alpha \int_{0}^{\infty} I_{n}(x) \eta(x) d x, n \geq 2(13) \\
R_{n}(0)= & \alpha \int_{0}^{\infty} W_{n}(x) \mu(x) d x, n \geq 0
\end{aligned}
$$

The normalizing condition is

$$
\begin{array}{r}
I_{0}+\sum_{n=1}^{\infty} \int_{0}^{\infty} I_{n}(x) d x+\sum_{n=0}^{\infty} \int_{0}^{\infty} W_{n}(x) d x+\sum_{n=1}^{\infty} \int_{0}^{\infty} R_{n}(x) d x \\
+\sum_{n=0}^{\infty} \int_{0}^{\infty} V_{n}(x) d x=1
\end{array}
$$

Define the probability generating functions

$$
\begin{aligned}
& I(x, z)=\sum_{n=1}^{\infty} \mathrm{I}_{\mathrm{n}}(x) z^{n} ; \quad W(x, z)=\sum_{n=0}^{\infty} W_{\mathrm{n}}(x) z^{n} \\
& R(x, z)=\sum_{n=1}^{\infty} R_{\mathrm{n}}(x) z^{n} \text { and } V(x, z)=\sum_{n=0}^{\infty} V_{\mathrm{n}}(x) z^{n}
\end{aligned}
$$

The following theorem discusses the steady state distribution of the system.

Theorem 2.

$$
\begin{aligned}
& \text { If } \\
& \lambda\left(\alpha \beta \mathrm{v}_{1}+\alpha \mathrm{b}_{1}+\overline{\alpha g}_{1}(1-\theta \beta)\right)+\bar{\alpha}<A *(\lambda)+\theta \beta\left(1-A^{*}(\lambda)\right) \\
& \text { then }
\end{aligned}
$$


The probability generating function of the orbit size when the server is idle is

$I(z)=\left(1-A^{*}(\lambda)\right)(((1-\beta) B *(\lambda-\lambda z)+(1-\theta) \beta$

$$
\begin{gathered}
B *(\lambda-\lambda z) V *(\lambda-\lambda z) \alpha z)+(z-\theta \beta B *(\lambda-\lambda z) \\
\left.V *(\lambda-\lambda z))\left(\alpha z G^{*}(\lambda-\lambda z)-1\right)\right) I_{0} / D(z)
\end{gathered}
$$

The probability generating function of the orbit size when the server is busy is

$$
W(z)=\alpha A *(\lambda)(B *(\lambda-\lambda z)-1) I_{0} / D(z)
$$

The probability generating function of the orbit size when the server is under repair is

$$
\begin{array}{r}
R(z)=\bar{\alpha} A *(\lambda)(z-\theta \beta B *(\lambda-\lambda z) V *(\lambda-\lambda z)) \\
(G *(\lambda-\lambda z)-1) I_{0} / D(z)
\end{array}
$$

The probability generating function of the orbit size when the server is on vacation is

$V(z)=\alpha \beta A^{*}(\lambda) B^{*}(\lambda-\lambda z)(V *(\lambda-\lambda z)-1) I_{0} / D(z)(19)$

The steady state probability that the system is empty is

$$
\begin{array}{r}
I_{0}=\left((1-\theta \beta)\left(A *(\lambda)-\alpha \lambda g_{1}-1\right)+\alpha-\right. \\
\left.\alpha \lambda\left(b_{1}+\beta v_{1}\right)\right) / \alpha A *(\lambda)
\end{array}
$$

where

$$
\begin{aligned}
D(z)= & z-\{\alpha(z+(1-z) A *(\lambda))[(1-\beta)+(1-\theta) \beta V *(\lambda-\lambda z)] \\
& B *(\lambda-\lambda z)-\theta \beta B *(\lambda-\lambda z) V *(\lambda-\lambda z)[1-\alpha G *(\lambda-\lambda z) \\
& (z+(1-z) A *(\lambda))]-z \bar{\alpha}(z+(1-z) A *(\lambda)) G *(\lambda-\lambda z)\}
\end{aligned}
$$

\section{Proof:}

Multiplying equations (1) - (14) by $\mathrm{z}^{\mathrm{n}}$ and summing over $\mathrm{n}, \mathrm{n}=0,1,2,3 \ldots$,we obtain the partial differential equations

$$
\begin{aligned}
& {\left[\frac{d}{d x}+\lambda+\eta(x)\right] I(x, z)=0} \\
& {\left[\frac{d}{d x}+\lambda(1-\mathrm{z})+\mu(x)\right] W(x, z)=0} \\
& {\left[\frac{d}{d x}+\lambda(1-\mathrm{z})+r(x)\right] R(x, z)=0} \\
& {\left[\frac{d}{d x}+\lambda(1-\mathrm{z})+\gamma(x)\right] V(x, z)=0}
\end{aligned}
$$

$I(0, z)=(1-\beta) \int_{0}^{\infty} W(x, z) \mu(x) d x+(1-\theta) \int_{0}^{\infty} V(x, z) \gamma(x) d x$

$$
+\int_{0}^{\infty} R(x, z) r(x) d x-\lambda I_{0}
$$

$W(0, z)=\alpha \lambda I_{0}+\alpha \lambda \int_{0}^{\infty} I(x, z) d x+\frac{\alpha}{z} \int_{0}^{\infty} I(x, z) \eta(x) d x$

$$
+\frac{\theta}{z} \int_{0}^{\infty} V(x, z) \gamma(x) d x
$$

$$
R(0, z)=\bar{\alpha} \lambda z \int_{0}^{\infty} I(x, z) d x+\alpha \int_{0}^{-\infty} I(x, z) \eta(x) d x+\bar{\alpha} \lambda z I_{0}
$$

$V(0, z)=\beta \int_{0}^{\infty} W(x, z) \mu(x) d x$

Solving the partial differential equations (21) - (24), we obtain

$$
\begin{aligned}
& I(x, z)=I(0, z) e^{-\lambda x}(1-A(x)) \\
& W(x, z)=W(0, z) e^{-\lambda(1-z) x}(1-B(x)) \\
& R(x, z)=R(0, z) e^{-\lambda(1-z) x}(1-G(x)) \\
& V(x, z)=V(0, z) e^{-\lambda(1-z) x}(1-V(x))
\end{aligned}
$$

Using equations (29) - (32) in equations (25) - (28) and after some algebraic manipulation, we get

$$
\begin{aligned}
& I(0, z)=(((1-\beta) B *(\lambda-\lambda z)+(1-\theta) \beta B *(\lambda-\lambda z) \\
&V *(\lambda-\lambda z) \alpha z)+(z-\theta \beta B *(\lambda-\lambda z) V *(\lambda-\lambda z)) \\
&\left.-\left(\alpha z G^{*}(\lambda-\lambda z)-1\right)\right) \lambda I_{0} / D(z) \\
& W(0, z)= \alpha A *(\lambda)(z-1) \lambda I_{0} / D(z) \\
& R(0, z)=-\alpha A *(\lambda)(z-\theta \beta B *(\lambda-\lambda z) V *(\lambda-\lambda z) \\
&(z-1) \lambda I_{0} / D(z)
\end{aligned}
$$

$V(0, z)=\alpha \beta A *(\lambda) B *(\lambda-\lambda z)(z-1) \lambda I_{0} / D(z)$

By substituting the expressions (33) to (36) in equations (29) to (32), we get the expressions for $I(x, z), W(x, z), R(x, z), V(x, z)$. Integrating these with respect to $\mathrm{x}$ from 0 to $\infty$, we obtain the equations (16) to (19). Using normalizing condition $I_{0}+I(1)+W(1)+R(1)+V(1)=1$ and solving we get the analytical expression for $I_{0}$.

\section{Corollary 1.}

The steady state probabilities of the server being idle, busy, repair and on vacation are

$$
\begin{array}{r}
I=I(1)=\left(1-A^{*}(\lambda)\right)\left(\bar{\alpha}-\theta \beta+\lambda\left(\alpha \beta v_{1}+\alpha b_{1}+\right.\right. \\
- \\
\left.\left.\alpha g_{1}(1-\theta \beta)\right)\right) / \alpha A^{*}(\lambda)
\end{array}
$$

$W=W(1)=\lambda b_{1}$

$R=R(1)=\bar{\alpha}(1-\theta \beta) \lambda g_{1}$ and

$V=V(1)=\beta \lambda v_{1}$.

\section{Corollary 2.}

The mean number of customers in the orbit and system under steady state conditions are

$$
\begin{aligned}
& \mathrm{L}_{\mathrm{q}}= N^{\prime} D^{\prime \prime} / 2 D^{\prime 2} \text { and } \mathrm{L}_{\mathrm{s}}=\mathrm{L}_{\mathrm{q}}+\lambda b_{1} \\
& \text { where } N^{\prime}=\alpha A^{*}(\lambda) I_{0}, \\
& \begin{aligned}
D^{\prime}= & (1-\theta \beta)\left(A^{*}(\lambda)-\bar{\alpha} \lambda g_{1}-1\right)+\alpha-\alpha \lambda\left(b_{1}+\beta v_{1}\right) \\
D^{\prime \prime}= & 2\left(1-A^{*}(\lambda)\right)\left[\lambda b_{1}(\alpha-\theta \beta)+\beta \lambda v_{1}(\alpha-\theta)+\right. \\
& \left.-\bar{\alpha}\left(1+\lambda g_{1}(1-\theta \beta)\right)\right]+\alpha \beta\left(2 \lambda^{2} b_{1} v_{1}+\lambda^{2} v_{2}\right)+ \\
& -{ }^{2} g_{1}\left(1-\theta \beta\left(\lambda v_{1}+\lambda b_{1}\right)\right)+\lambda^{2} b_{2}(1-\alpha \theta \beta)
\end{aligned}
\end{aligned}
$$


Proof :

The probability generating function of the number of customers in the orbit is $H(z)=I_{0}+I(z)+W(z)+R(Z)+V(z)$

$$
\mathrm{L}_{\mathrm{q}}=\lim _{z \rightarrow 1} \frac{d}{d z} H(z)
$$

Similarly, the probability generating function of the number of customers in the system is $K(z)=I_{0}+I(z)+z W(z)+R(Z)+V(z)$

$$
\mathrm{L}_{\mathrm{s}}=\lim _{z \rightarrow 1} \frac{d}{d z} K(z)
$$

Theorem 3.

The steady state availability of the server is

$$
\begin{aligned}
A & =I_{0}+I(1)+W(1) \\
& =1-\lambda \beta v_{1}-\bar{\alpha} \lambda g_{1}(1-\theta \beta) / \alpha .
\end{aligned}
$$

\section{PARTICULAR CASES}

If $\alpha=1$, the model under study reduces to M/G/1 retrial queue with vacation and orbital search.

If $\beta=1$, the model reduces to $\mathrm{M} / \mathrm{G} / 1$ retrial queue with starting failure and orbital search.

If $\theta=0$, the model under study reduces to $\mathrm{M} / \mathrm{G} / 1$ retrial queue with vacation and starting failure.

If $A^{*}(\lambda) \rightarrow 1$, the system reduces to $M / G / 1$ queue with vacation, starting failure and orbital search.

\section{NUMERICAL RESULTS}

The numerical calculations are carried out by assuming the service time, retrial time, repair time and vacation time to follow exponential distribution with respective parameters $\mu, \eta, r$ and $\gamma$. As expected it is observed that

- $I_{0}$ increases with increase in $\beta, \theta, \alpha, \mu, \eta$ and $r$.

- $\quad I, \mathrm{~L}_{\mathrm{s}}$ and $\mathrm{L}_{\mathrm{q}}$ decrease with increase in $\mu, \theta, \alpha$ and $\eta$ and increase with increase in $\beta$.

- Increase in $\lambda$ increases $I, W, R, V, \mathrm{~L}_{\mathrm{s}}$ and $\mathrm{L}_{\mathrm{q}}$.

Table 1: Effect of $\lambda$ on the performance measures $(\beta=\theta=\alpha=0.5, \mu=r=\gamma=10$ and $\eta=6)$.

\begin{tabular}{|c|c|c|c|c|c|c|c|}
\hline$\lambda$ & $\mathrm{I}_{0}$ & $\mathrm{I}$ & $\mathrm{W}$ & $\mathrm{R}$ & $\mathrm{V}$ & $\mathrm{L}_{\mathrm{s}}$ & $\mathrm{L}_{\mathrm{q}}$ \\
\hline 1.2000 & 0.5760 & 0.0630 & 0.1200 & 0.0900 & 0.0600 & 0.2618 & 0.1418 \\
\hline 1.4000 & 0.4948 & 0.0828 & 0.1400 & 0.1050 & 0.0700 & 0.3329 & 0.1929 \\
\hline 1.6000 & 0.4107 & 0.1053 & 0.1600 & 0.1200 & 0.0800 & 0.4260 & 0.2660 \\
\hline 1.8000 & 0.3235 & 0.1305 & 0.1800 & 0.1350 & 0.0900 & 0.5601 & 0.3801 \\
\hline 2.0000 & 0.2333 & 0.1583 & 0.2000 & 0.1500 & 0.1000 & 0.7857 & 0.5857 \\
\hline
\end{tabular}

Table 2 : Effect of $\mu$ on the performance measures $(\lambda=1, \beta=\theta=\alpha=0.5, r=\gamma=10$ and $\eta=6)$

\begin{tabular}{|c|c|c|c|c|c|c|c|}
\hline$\mu$ & $\mathrm{I}_{0}$ & $\mathrm{I}$ & $\mathrm{W}$ & $\mathrm{R}$ & $\mathrm{V}$ & $\mathrm{L}_{\mathrm{s}}$ & $\mathrm{L}_{\mathrm{q}}$ \\
\hline 15.0000 & 0.6931 & 0.0403 & 0.0667 & 0.0750 & 0.0500 & 0.1728 & 0.1061 \\
\hline 20.0000 & 0.7125 & 0.0375 & 0.0500 & 0.0750 & 0.0500 & 0.1548 & 0.1048 \\
\hline 25.0000 & 0.7242 & 0.0358 & 0.0400 & 0.0750 & 0.0500 & 0.1433 & 0.1033 \\
\hline 30.0000 & 0.7319 & 0.0347 & 0.0333 & 0.0750 & 0.0500 & 0.1354 & 0.1020 \\
\hline 35.0000 & 0.7375 & 0.0339 & 0.0286 & 0.0750 & 0.0500 & 0.1295 & 0.1010 \\
\hline
\end{tabular}

Table 3: Effect of $\boldsymbol{\eta}$ on the performance measures $(\lambda=1, \beta=\theta=\alpha=0.5, \mu=r=\gamma=10)$

\begin{tabular}{|c|c|c|c|c|c|c|c|}
\hline$\eta$ & $\mathrm{I}_{0}$ & $\mathrm{I}$ & $\mathrm{W}$ & $\mathrm{R}$ & $\mathrm{V}$ & $\mathrm{L}_{\mathrm{s}}$ & $\mathrm{L}_{\mathrm{q}}$ \\
\hline 1.2000 & 0.1708 & 0.2292 & 0.1000 & 0.0750 & 0.0500 & 4.4878 & 4.3878 \\
\hline 1.4000 & 0.2571 & 0.1964 & 0.1000 & 0.0750 & 0.0500 & 2.5417 & 2.4417 \\
\hline 1.6000 & 0.3219 & 0.1719 & 0.1000 & 0.0750 & 0.0500 & 1.7670 & 1.6670 \\
\hline 1.8000 & 0.3722 & 0.1528 & 0.1000 & 0.0750 & 0.0500 & 1.3507 & 1.2507 \\
\hline 2.0000 & 0.4125 & 0.1375 & 0.1000 & 0.0750 & 0.0500 & 1.0909 & 0.9909 \\
\hline
\end{tabular}

Table 4: Effect of $r$ on the performance measures $(\lambda=1, \beta=\theta=\alpha=0.5, \mu=\gamma=10$ and $\eta=6$ )

\begin{tabular}{|c|c|c|c|c|c|c|c|}
\hline$r$ & $\mathrm{I}_{0}$ & $\mathrm{I}$ & $\mathrm{W}$ & $\mathrm{R}$ & $\mathrm{V}$ & $\mathrm{L}_{\mathrm{s}}$ & $\mathrm{L}_{\mathrm{q}}$ \\
\hline 15.0000 & 0.6833 & 0.0417 & 0.1000 & 0.0500 & 0.0500 & 0.2474 & 0.1474 \\
\hline 20.0000 & 0.6979 & 0.0396 & 0.1000 & 0.0375 & 0.0500 & 0.2678 & 0.1678 \\
\hline 25.0000 & 0.7067 & 0.0383 & 0.1000 & 0.0300 & 0.0500 & 0.2796 & 0.1796 \\
\hline 30.0000 & 0.7125 & 0.0375 & 0.1000 & 0.0250 & 0.0500 & 0.2873 & 0.1873 \\
\hline 35.0000 & 0.7167 & 0.0369 & 0.1000 & 0.0214 & 0.0500 & 0.2928 & 0.1928 \\
\hline
\end{tabular}

Table 5: Effect of $\boldsymbol{\theta}$ on the performance measures $(\lambda=1, \beta=\alpha=0.5, \mu=r=\gamma=10$ and $\eta=6)$

\begin{tabular}{|c|c|c|c|c|c|c|c|}
\hline$\theta$ & $\mathrm{I}_{0}$ & $\mathrm{I}$ & $\mathrm{W}$ & $\mathrm{R}$ & $\mathrm{V}$ & $\mathrm{L}_{\mathrm{s}}$ & $\mathrm{L}_{\mathrm{q}}$ \\
\hline 0.2000 & 0.5867 & 0.0483 & 0.1000 & 0.0900 & 0.0500 & 0.2281 & 0.1281 \\
\hline 0.4000 & 0.6317 & 0.0467 & 0.1000 & 0.0800 & 0.0500 & 0.2113 & 0.1113 \\
\hline 0.6000 & 0.6767 & 0.0450 & 0.1000 & 0.0700 & 0.0500 & 0.1968 & 0.0968 \\
\hline 0.8000 & 0.7217 & 0.0433 & 0.1000 & 0.0600 & 0.0500 & 0.1841 & 0.0841 \\
\hline 1.0000 & 0.7667 & 0.0417 & 0.1000 & 0.0500 & 0.0500 & 0.1728 & 0.0728 \\
\hline
\end{tabular}

Table 6: Effect of $\boldsymbol{\beta}$ on the performance measures $(\lambda=1, \theta=\alpha=0.5, \mu=r=\gamma=10$ and $\eta=6)$

\begin{tabular}{|c|c|c|c|c|c|c|c|}
\hline$\beta$ & $\mathrm{I}_{0}$ & $\mathrm{I}$ & $\mathrm{W}$ & $\mathrm{R}$ & $\mathrm{V}$ & $\mathrm{L}_{\mathrm{s}}$ & $\mathrm{L}_{\mathrm{q}}$ \\
\hline 0.2000 & 0.6217 & 0.0383 & 0.1000 & 0.0900 & 0.0200 & 0.2016 & 0.1016 \\
\hline 0.4000 & 0.6433 & 0.0433 & 0.1000 & 0.0800 & 0.0400 & 0.2031 & 0.1031 \\
\hline 0.6000 & 0.6650 & 0.0483 & 0.1000 & 0.0700 & 0.0600 & 0.2045 & 0.1045 \\
\hline 0.8000 & 0.6867 & 0.0533 & 0.1000 & 0.0600 & 0.0800 & 0.2058 & 0.1058 \\
\hline 1.0000 & 0.7083 & 0.0583 & 0.1000 & 0.0500 & 0.1000 & 0.2071 & 0.1071 \\
\hline
\end{tabular}


Table 7: Effect of $\alpha$ on the performance measures

$(\lambda=1, \beta=\theta=0.5, \mu=r=\gamma=10$ and $\eta=6)$

\begin{tabular}{|c|c|c|c|c|c|c|c|}
\hline$\alpha$ & $\mathrm{I}_{0}$ & $\mathrm{I}$ & $\mathrm{W}$ & $\mathrm{R}$ & $\mathrm{V}$ & $\mathrm{L}_{\mathrm{s}}$ & $\mathrm{L}_{\mathrm{q}}$ \\
\hline 0.2000 & 0.0167 & 0.1083 & 0.1000 & 0.3000 & 0.0500 & 13.4000 & 13.3000 \\
\hline 0.4000 & 0.5479 & 0.0563 & 0.1000 & 0.1125 & 0.0500 & 0.2707 & 0.1707 \\
\hline 0.6000 & 0.7250 & 0.0389 & 0.1000 & 0.0500 & 0.0500 & 0.1701 & 0.0701 \\
\hline 0.8000 & 0.8135 & 0.0302 & 0.1000 & 0.0187 & 0.0500 & 0.1362 & 0.0362 \\
\hline 1.0000 & 0.8667 & 0.0250 & 0.1000 & 0.0000 & 0.0500 & 0.1192 & 0.0192 \\
\hline
\end{tabular}

\section{REFERENCES}

[1] Artalejo, J.R., Joshua, V.C. and Krishnamoorthy, A., 2002. An M/G/1 Retrial Queue with orbital search by the server, In Advances in stochastic modeling eds., New Jersey, Notable Publications, 41 - 54.

[2] Baba, Y., 2005. Analysis of a GI/M/1 queue with multiple working vacations. Operations Research Letters, 33(2), 201-209.

[3] Doshi, B.T., 1990. Single-server Queues with Vacations, Stochastic Analysis of Computer and Communications Systems, $217-265$.

[4] Ke, J. C., 2003. The optimal control of an M/G/1 queueing system with server vacations, startup and breakdowns, Computers and Industrial Engineering, 44(4), 567-579.
[5] Ke, J. C. and Chang, F. M., 2009. $\mathrm{M}^{\mathrm{x}} / \mathrm{G}_{1}, \mathrm{G}_{2} / 1$ Retrial Queue with Bernoulli Vacation Schedules with Repeated Attempts and Starting Failures, Applied Mathematical Modeling, 33, 3186-3196.

[6] Krishnakumar, B., Pavai Madheswari, S. and Vijayakumar, A., 2002. The M/G/1 retrial queue with feedback and starting failures, Applied Mathematical Modelling, Volume 26, 1057-1075.

[7] Mokaddis, G. S., Metwally, S. A. and Zaki, B. M., 2007. A Feedback Retrial Queuing System with Starting Failures and Single Vacation, Tamkang Journal of Science and Engineering, Vol. 10, N0. 3, 183-192.

[8] Sennot, L.A., Humblet, P.A. and Tweedie, R.L., 1983 Mean drifts and non-ergodicity of Markov chains, Operations Research, 31, 783-789.

[9] Takagi, H., 1991. Queueing Analysis: Vacation and Priority Systems, North-Holland, Amsterdam, Vol. 1. 230

[10] $\mathrm{Wu}, \mathrm{D}$. A. and Takagi, H., 2005. M/G/1 queue with multiple working vacations. Performance Evaluation, 63 , 654-681.

[11] Yang, T. and Li, H., 1994. The M/G/1 Retrial Queue with the Server subjected to Starting Failure, Queueing System, 16, 83-96. 